\section{Abstractions}

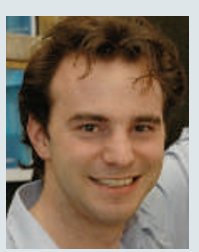

PENULTIMATE AUTHOR

Quantum mechanics often conflicts with everyday assumptions. Entanglement, for example, defies the idea that two objects cannot physically interact with one another at a distance. Physicists have tried to resolve this by abandoning the idea of 'locality' in their theories. But as Markus Aspelmeyer from the University of Vienna in Austria and his colleagues reveal on page 871 , this is not enough and certain aspects of realism which holds that reality exists independently of observers - must also be cast aside.

Why is quantum physics so difficult for most people to understand?

Because classical concepts cannot be used to explain quantum phenomena.

\section{What attracts people to the field?} We want to find out why quantum theory works so perfectly and yet cannot be understood by the intuitive concepts of the other physical theories. There are observable phenomena that cannot be explained by our current 'world view'. Isn't that attractive?

What is the difference between reality in normal life and from a quantum perspective? People observe outcomes and believe each observed event can be fully explained by the properties of the system and independently of the observation. In this case, things have 'real' properties. This conclusion is wrong for the quantum world. Quantum physics does not tell us how nature is, only what we can say about nature. And we can only make statements about probabilities for outcomes.

What consequences do your findings have for the development of quantum theory? I don't know. But anybody formulating a non-local hidden-variable theory will have to specify their exact idea of realism.

What are the largest and smallest systems to which your research can be applied? In principle, quantum theory does not stop at massive objects. Quantum behaviour has been demonstrated for massive objects up to the size of macromolecules. It would be interesting to extend this work to even larger masses and sizes.

Did your studies in philosophy help your understanding of quantum mechanics? I think they made me more sensitive to the underlying structures and assumptions of theories. You need to know the preconditions under which you are able to make statements about the physical world and observations in the physical world.

Is every quantum mechanic a philosopher? I think so. But not many would admit it.
MAKING THE PAPER

Liane Young \& Michael Koenigs

\section{Brain damage offers clues to the role of emotion in moral decisions.}

When faced with a moral dilemma, are the decisions people make guided by their emotions or rational arguments? A number of studies have suggested that emotions are important for making moral judgments but, according to Ralph Adolphs of the California Institute of Technology in Pasadena, these conclusions have all been based on correlations. About four years ago, he and his colleagues began talking about ways to demonstrate a direct link between emotions and morality.

"We had been thinking about experiments and even gathered some preliminary data, but the project really needed someone to take charge and push it through," says Adolphs. Graduate students Liane Young in Marc Hauser's lab at Harvard University in Cambridge, Massachusetts, and Michael Koenigs from Daniel Tranel's lab at the University of Iowa in Iowa City took up the challenge.

"We thought it would be interesting to show what happens to moral judgment if emotions are taken out of the picture," says Young. To do this, she and Koenigs turned to a region of the brain known as the ventromedial "Emotion is prefrontal cortex (VMPC).

Individuals with lesions in the VMPC have reduced social emotions, such as compassion, shame and guilt - in other words, the types of emotion an individual might use to make moral judgments. People with VMPC damage are of normal intelligence and can needed with personal choices that involve a high degree of conflict." understand and remember social and moral norms. In addition, a recent functional imaging study showed that the VMPC is activated when individuals are asked to make moral decisions. On the basis of these results, the team quickly found six patients with damage to the VMPC.
They presented patients and controls with moral situations requiring them to choose between two courses of action. They found controls only made different choices from VMPC patients when presented with certain scenarios. Those that elicited different responses - presumably those in which emotions have a role involved a 'personal' component. For example, when faced with the option of pushing someone off a bridge to prevent a runaway boxcart from hitting five people, normal individuals have to overcome a 'personal' response against doing harm to an individual in order to agree with the utilitarian, or more pragmatic, response. People with VMPC damage made the utilitarian decision much more frequently, although not in every instance (see page 908).

The distinction between the two groups only occurred when individuals were faced with what were considered 'gut-wrenching' dilemmas. For example, when given the choice of killing their own child to save money the VMPC patients made the same decision - not to kill the child - as normal individuals. But if the scenario was to smother their own child to prevent harm to others, the VMPC patients would more often go for the utilitarian choice. So for certain moral dilemmas, the team concludes, a functional VMPC is needed for 'normal' judgement of 'right' and 'wrong'.

"We found that emotion is needed with personal choices that involve a high degree of conflict," says Adolphs. The result opens up many questions to investigation. It is not clear, for example, how much emotion plays a part in different moral judgments. To address this, researchers would have to find a way to actually measure the amount of emotional response elicited by different moral judgments, which Young and Koenigs tried to do, but without success. Another limitation of the study is that the moral choices they used were artificial, life-and-death situations. "It would be interesting to see how results might vary if people were faced with more regular moral judgments," says Koenigs.

\title{
KEY INSPIRATION
}

Carbohydrates are essential

for many biological processes, but their molecular complexity means that synthesizing them requires "intermittent, tedious work-ups and timeconsuming purifications", says Shang-Cheng Hung, a chemistry professor at the National Tsing Hua University in Hsinchu, Taiwan. Simplifying the process would make it easier to produce hundreds, if not thousands, of these useful biopolymers.
Hung drew on two earlier mentors' work to find chemical shortcuts (see page 896). He adapted a concept developed by his former postdoc adviser Chi-Huey Wong, who is now president of Academia Sinica in Taipei. Wong's 'one-pot' concept allows many chemical building blocks to be produced at once. Hung then modified a technique devised by his $\mathrm{PhD}$ supervisor Chun-Chen Liao, a professor at National Tsing Hua University. His technique allows a single catalyst to drive reactions at relatively low temperatures. "When we combined both processes, we obtained excellent selectivity and good yields," Hung says. Hung started the project seven years ago, but needed time to find optimal reaction conditions by trial and error. His mentors' inspiration and his group's efforts should make carbohydrate synthesis faster and easier for the next wave of chemists. 